\title{
Anxiolytic Medication and Long Acting Injectable Antipsychotics (LAI) in Schizophrenia: A Post-hoc Analysis of a 3-year Prospective Cohort Study Carla Spínola ${ }^{1}$, Daniel Neto ${ }^{2 *}$, Leonor Santana ${ }^{3}$ and Joaquim Gago $^{4}$
}

${ }^{1}$ Psychiatrist, Serviço de Psiquiatria do Serviço de Saúde da Região Autónoma da Madeira, Portugal

${ }^{2}$ Psychiatrist, Casa de Saúde São João de Deus, Funchal, Portugal

${ }^{3}$ Psychiatry Resident, Centro Hospitalar de Lisboa Ocidental, EPE, Portugal

${ }^{4}$ Senior Psychiatrist Consultant, Centro Hospitalar de Lisboa Ocidental, EPE, Portugal

\section{Abstract}

Schizophrenia is a disabling disease with a significant proportion of patients having more than one episode of acute psychotic symptoms. The main reason for a relapse is non-adherence, followed by age of onset. Anxiety in schizophrenia has not been thoroughly investigated; however it is clinically present in more than $40 \%$ of patients as a comorbidity.

The authors did a post-hoc analysis of a three-year prospective cohort study in a community mental health team (CMHT) in Portugal. We included all patients with the diagnosis of schizophrenia, aged 18 years and above, followed during 2015. Parametric tests ( $\chi^{2}$ test, student $t$ test) were used for statistical analysis according to the variable type. The pharmacological treatment prescribed was screened during this period, with particular interest in the prescription of anxiolytic medication and long-acting injectable antipsychotics (LAI). We categorized the LAI available in Portugal according to the Neuroscience based Nomenclature $(\mathrm{NbN})[1]$.

A total of 145 patients were selected. We found a high rate of polypharmacy. Our primary outcome was hospital admissions. Patients included in the oral antipsychotic group had fewer admissions ( $\mathrm{p}$-value 0.043 ) and a shorter duration of hospitalization ( $\mathrm{p}$-value 0.041 ). Only duration of illness ( $\mathrm{p}$-value $<0.001$ ) and being prescribed with anxiolytics (odds-ratio 2.8; p-value 0.015 ) at baseline were associated with a higher number of hospital admissions.

In the LAI group, we found a substantial prescription of different oral antipsychotics. The prescription of anxiolytics at baseline showed a negative association with hospital admissions. Larger and multicentric prospective cohorts are needed to clarify the effects of specific classes of antipsychotics in the long-term prevention of admissions, symptomatology and remission. Anxiety and anxiolytics should be further investigated in schizophrenia.

\section{Background}

Schizophrenia is a very disabling disease with a significant proportion of patients having more than one episode of acute psychotic symptoms [2]. The main reason for a relapse is nonadherence, followed by age of onset, justifying the use of long acting injectable antipsychotics (LAI) [3].

Atypical antipsychotics have been associated with a lower risk of extrapyramidal symptoms but a higher risk of metabolic adverse effects. They became the first line therapy in the last few years and it is suggested that atypical LAI could outperform oral medication [4]. Despite the prescription of LAI, patients still relapse, having new psychotic episodes. In the last few years there is growing evidence regarding the recognition of subsyndromal symptoms, as they may be important to prevent further escalation of psychotic symptoms and re-admissions.

According to previous studies, anxiety was found in $41.5 \%$ of patients with schizophrenia. Patients with anxiety symptoms evidence higher functional impairment, mainly in the professional and social functioning. The group of patients with comorbid anxiety disorders showed higher positive symptoms and were more often under treatment with antianxiety drugs. Contrasting, they had lower number of hospitalizations [5]. Huppert et al. also showed that quality of life was also affected by the presence of anxiety and depression.
Particularly, anxiety was associated with bigger difficulties in daily life activities such as family and social activities, despite the control of positive symptoms [6].

Antidepressant and anxiolytic medication are frequently used as co-adjuvant therapy in schizophrenia. The CATIE trial showed that at baseline $38 \%$ of patients were prescribed antidepressants and $22 \%$ anxiolytics. There was an association with the presence of anxiety or depression, being female, and being treated with second-generation antipsychotics. Depressive mood showed only partial response with this approach [7].

At follow-up in the same trial, a higher positive symptom score and younger age were associated in an earlier prescription of antidepressants. The duration of antidepressant treatment was longer in patients with lower education and in those with a history of alcohol

*Corresponding Author: Dr. Daniel Neto, Psychiatrist, Casa de Saúde São João de Deus, Funchal, Portugal; E-mail: danielcarvalhoneto@gmail.com

Citation: Spínola C, Neto D, Santana L, Gago J (2021) Anxiolytic Medication and Long Acting Injectable Antipsychotics (LAI) in Schizophrenia: A Post-hoc Analysis of a 3-year Prospective Cohort Study. Int J Psychol Behav Anal 7: 172. doi: https:// doi.org/10.15344/2455-3867/2021/172

Copyright: (C) 2021 Spínola et al. This is an open-access article distributed under the terms of the Creative Commons Attribution License, which permits unrestricted use, distribution, and reproduction in any medium, provided the original author and source are credited. 
Citation: Spínola C, Neto D, Santana L, Gago J (2021) Anxiolytic Medication and Long Acting Injectable Antipsychotics (LAI) in Schizophrenia: A Post-hoc Analysis of a 3-year Prospective Cohort Study. Int J Psychol Behav Anal 7: 172. doi: https://doi.org/10.15344/2455-3867/2021/172

Page 2 of 4

use disorder. Predictors of anxiolytic initiation were being separated/ divorced, not being African-American, younger age, higher body mass index and akathisia [8].

Ritsner et al. showed that patients with schizophrenia treated with antidepressants and anxiolytics have better quality of life [9].

Regardless of these findings, some evidence shows a 4.8 -fold higher mortality risk with the chronic use of anxiolytics (including cardiovascular and suicide-related mortality). This could be associated with the illness severity or comorbid anxiety. An increased risk for accidents due to daytime sedation or cognitive impairment is another hypothesis [10]. Particularly, the deleterious cognitive effect was suggested to be associated with impaired attention/working memory, independently of socio-demographic variables and illness characteristics [11]

Our aim is to study the possible association between hospital admissions and emergency department visits among schizophrenic patients who had been prescribed anxiolytic medication. We compared two groups, considering oral vs long-acting injectables and concomitant medication.

\section{Methods}

We conducted a post-hoc analysis of a three-year prospective cohort study in a community mental health team (CMHT) in Portugal, urban area. We included all patients with the diagnosis of schizophrenia according to the International Classification of Disorders, version 10th, published by the World Health Organization, aged 18 years and above and followed during 2015 in the CMHT. The study was approved by the ethical committee of our hospital.

Several demographic and clinical baseline characteristics were recorded, and the primary outcome was psychiatric admission. We included emergency department visits (ED) and occupational status as secondary outcomes.

Parametric tests ( $\chi^{2}$ test, student $t$ test) were used for statistical analysis according to the variable type. Statistical significance was set at 0.05 .

We categorized the antipsychotics available in Portugal according to the Neuroscience based Nomenclature $(\mathrm{NbN}) 1$ : Receptor antagonist (RA) (D2) amisulpride, haloperidol, fluphenazine, pimozide, sulpiride, e zuclopenthixol; RA (D2, 5-HT2) cyamemazine, chlorpromazine; levomepromazine, flupentixol, melperone, olanzapine, ziprasidone, zotepine; RA (D2, 5-HT2, NE alpha-2) clozapine, paliperidone e risperidone; Receptor partial agonist (RpA) (D2, 5-HT1A) and RA (5-HT2A) aripiprazole; RA (D2, 5-HT2) and reuptake inhibitor (NET) quetiapine.

\section{Results}

According to our selection criteria, 145 patients were selected. In the early follow-up period, we had 15 dropouts mainly because of change of address. In the remaining group (130 patients), $62.8 \%$ were males, with a mean age of 47 years (standard-deviation [SD] of 12.9 years), mainly unemployed (37.7\%) or retired due to disability (20.7\%), with a mean duration of illness of 8.7 years (SD 3.1 years).

At baseline, $48.5 \%$ were prescribed with one oral antipsychotic (OA). In the group of patients taking LAI, $94 \%$ were additionally prescribed with OA. In the group of patients taking only OA, we found that $41 \%$ were prescribed with more than one antipsychotic. Mood stabilizers were prescribed in $21.5 \%$ patients, antidepressants in $40 \%$, anxiolytics in $50 \%$ and hypnotics in $13.1 \%$.

Table 1 presents the social-demographic and main differences between the two groups: OA and LAI. We found a higher prescription of antipsychotic polypharmacy in the LAI group, which was statistically significant ( $\mathrm{p}$-value $<0.001)$, mainly regarding the receptor antagonist D2 class ( $\mathrm{p}$-value $<0.001$ ), receptor antagonist (D2, 5-HT2) and reuptake inhibitor (NET) class (p-value 0.020).

\begin{tabular}{|l|l|l|l|}
\hline & OA & LAI & p-value \\
\hline $\mathrm{N}$ & 51 & 79 & \\
\hline Male & $31(61 \%)$ & $50(64 \%)$ & 0.70 \\
\hline Age, mean (SD) & $46.5(13)$ & $47.4(13)$ & 0.69 \\
\hline Duration of Illness, mean (SD) & $8.9(3.1)$ & $8.5(3.1)$ & 0.55 \\
\hline Antipsychotics (more than 1) & $21(41 \%)$ & $74(94 \%)$ & $<\mathbf{0 . 0 0 1}$ \\
\hline Oral antipsychotics (more than one) & $21(41 \%)$ & $40(51 \%)$ & 0.29 \\
\hline RA (D2) & $8(16 \%)$ & $40(51 \%)$ & $<\mathbf{0 . 0 0 1}$ \\
\hline RA (D2, 5-HT2) & $24(47 \%)$ & $31(39 \%)$ & 0.38 \\
\hline RA (D2, 5-HT2, NE alpha-2) & $24(47 \%)$ & $45(57 \%)$ & 0.27 \\
\hline $\begin{array}{l}\text { RpA (D2, 5-HT1A) and RA (5- } \\
\text { HT2A) }\end{array}$ & $7(14 \%)$ & $9(11 \%)$ & 0.69 \\
\hline $\begin{array}{l}\text { RA (D2, 5-HT2) and reuptake } \\
\text { inhibitor (NET) }\end{array}$ & $6(12 \%)$ & $23(29 \%)$ & $\mathbf{0 . 0 2 0}$ \\
\hline Mood stabilizers, mean (SD) & $0.3(0.5)$ & $0.2(0.5)$ & 0.60 \\
\hline Antidepressants, mean (SD) & $0.6(0.8)$ & $0.5(0.8)$ & 0.70 \\
\hline Anxiolytics, mean (SD) & $0.7(0.8)$ & $0.8(0.9)$ & 0.60 \\
\hline Hypnotics, mean (SD) & $0.2(0.5)$ & $0.1(0.4)$ & 0.62 \\
\hline
\end{tabular}

Table 1: Differences between OA and LAI groups.

RA: Receptorantagonist, RpA: Receptorpartialagonist.

In Table 2 we present the differences between oral medication and LAI classes according to $\mathrm{NbN}$. We found that the difference between groups regarding antipsychotic polypharmacy in the LAI group was statically significant $(\mathrm{p}$-value $<0.001)$, with almost all the patients taking LAI being prescribed with RA (D2) (p-value $<0.001$ ), RA (D2, 5-HT2, NE alpha-2) (p-value 0.008) and RpA (D2, 5-HT1A) and RA (5-HT2A) (p-value 0.004) classes.

The individual analyses showed that in the LAI group the use of antipsychotics is not restricted to their own class. The RA (D2) class (52 patients) had $69 \%$ use of OA of the same class, but also $42 \%$ of RA (D2, 5-HT2), 46\% of the RA (D2, 5-HT2, NE alpha-2) class, $8 \%$ of the RpA (D2, 5-HT1A) and RA (5-HT2A) class and 31\% of the RA (D2, 5-HT2) and reuptake inhibitor (NET) class. The RA (D2, 5 -HT2) class (2 patients) had 50\% prescribed of the RA (D2), RA (D2, 5-HT2) and RA (D2, 5-HT2) and reuptake inhibitor (NET) classes. The RA (D2, 5-HT2, NE alpha-2) class (23 patients) had prescribed $83 \%$ of the same class, and $13 \%$ of the RA (D2) class, $35 \%$ of the RA (D2, 5-HT2) class, $13 \%$ of the RpA (D2, 5-HT1A) and RA (5-HT2A) class and $22 \%$ of the RA (D2, 5-HT2) and reuptake inhibitor (NET) class. The RpA (D2, 5-HT1A) and RA (5-HT2A) class (2 patients), had $100 \%$ prescriptions from the RA (D2, 5-HT2, NE alpha-2) and RpA (D2, 5-HT1A) and RA (5-HT2A) classes and 50\% in RA (D2, 5 -HT2) and reuptake inhibitor (NET) class. 
Citation: Spínola C, Neto D, Santana L, Gago J (2021) Anxiolytic Medication and Long Acting Injectable Antipsychotics (LAI) in Schizophrenia: A Post-hoc Analysis of a 3-year Prospective Cohort Study. Int J Psychol Behav Anal 7: 172. doi: https://doi.org/10.15344/2455-3867/2021/172

Page 3 of 4

Our primary outcome was hospital admissions. Patients in the OA group had fewer admissions (p-value 0.043) and a shorter hospitalization (p-value 0.041) (Table 3). The Receptor antagonist (D2) LAI class was the closest to oral antipsychotics group (Table 4). Comparing the LAI RA (D2) with OA, there was no statistical significant difference ( $\mathrm{p}$-value 0.343). Comparing the LAI RA (D2, 5-HT2, NE alpha-2) with both OA and LAI RA (D2), there was a significant difference, disfavoring the LAI RA (D2, 5-HT2, NE alpha-2) class (p-value 0.003 and p-value 0.032 , respectively).

We analyzed known associations between baseline variables (age, sex, education, occupation, illness duration and medication) and hospital admissions. Only duration of illness ( $\mathrm{p}$-value $<0.001)$ and being prescribed with anxiolytics (odds-ratio 2.8; p-value 0.015 ) at baseline were associated with hospital admissions. Higher duration of illness showed a protective effect and anxiolytic prescription a negative association. Patients taking more than one antipsychotic were not associated with a higher number of admissions ( $\mathrm{p}$-value 0.778).

The secondary outcome was emergency department visits. We did not found any association with the prescription profile. Having a work occupation showed only a statistical trend ( $\mathrm{p}$-value 0.071 ), higher in the OA group.

\section{Discussion}

We present a real-world analysis of patients diagnosed with schizophrenia followed in a CMHT in an urban area of Portugal.

\begin{tabular}{|l|l|l|l|}
\hline & AO & LAI & p-value \\
\hline $\mathrm{N}$ & 51 & 79 & \\
\hline Hospital admissions, mean (SD) & $0.2(0.6)$ & $0.5(0.9)$ & $\mathbf{0 . 0 4 3}$ \\
\hline $\begin{array}{l}\text { Hospital admissions, days, } \\
\text { mean (SD) }\end{array}$ & $4.2(11.8)$ & $14.5(34.4)$ & $\mathbf{0 . 0 4 1}$ \\
\hline $\begin{array}{l}\text { Emergency Department visits, } \\
\text { mean (SD) }\end{array}$ & $2.6(1.8)$ & $2.6(1.9)$ & 0.98 \\
\hline Occupation (having work) & $11(21.6 \%)$ & $8(10.1 \%)$ & 0.071 \\
\hline
\end{tabular}

Table 3: Admission rate difference between OA and LAI groups.

Some authors argue that LAI can be used without any other antipsychotic, justifying their use to improve adherence [4]. In our study we found an association between antipsychotic polypharmacy and LAI prescription. This finding could suggest that more resistant disorders are treated with antipsychotic polypharmacy. According to this hypothesis, a higher number of hospital admissions was expected, but in our results, there was no difference in hospital admissions. We could argue that the polypharmacy came from the same class, but in the two major LAI classes, more than $50 \%$ of patients were taking oral medication with a different profile from the LAI classes.

According to these findings, we acknowledge that the monotherapy with a LAI antipsychotic may not be possible for a considerable number of patients, which contrast with internationally recognized clinical guidelines. In clinical practice, we discuss with patients that LAI could be a way to avoid oral medication but, according to our

\begin{tabular}{|l|l|l|l|l|l|l|}
\hline & oral only & RA (D2) & RA (D2, 5-HT2) & $\begin{array}{l}\text { RA (D2, 5-HT2, } \\
\text { NE alpha-2) }\end{array}$ & $\begin{array}{l}\text { RpA (D2, 5-HT1A) and } \\
\text { RA (5-HT2A) }\end{array}$ & p-value \\
\hline N & 51 & 52 & 2 & 23 & 2 & \\
\hline Male & $31(61 \%)$ & $36(71 \%)$ & $2(100 \%)$ & $11(48 \%)$ & $1(50 \%)$ & 0.29 \\
\hline Age, mean (SD) & $46.5(13)$ & $48.6(13.8)$ & $41(1.4)$ & $45.7(10)$ & $43.5(30.4)$ & 0.80 \\
\hline Duration of Illness, mean (SD) & $8.9(3.1)$ & $9.1(2.7)$ & $11(0)$ & $7.2(3.6)$ & $6.5(6.4)$ & 0.074 \\
\hline Antipsychotics (more than 1) & $21(41 \%)$ & $47(90 \%)$ & $2(100 \%)$ & $23(100 \%)$ & $2(100 \%)$ & $<0.001$ \\
\hline Oral antipsychotics(more than one) & $21(41 \%)$ & $26(50 \%)$ & $1(50 \%)$ & $11(48 \%)$ & $2(100 \%)$ & 0.53 \\
\hline RA (D2) & $8(16 \%)$ & $36(69 \%)$ & $1(50 \%)$ & $3(13 \%)$ & $0(0 \%)$ & $<$ \\
\hline RA (D2, 5-HT2) & $24(47 \%)$ & $22(42 \%)$ & $1(50 \%)$ & $8(35 \%)$ & $0(0 \%)$ & 0.64 \\
\hline RA (D2, 5-HT2, NE alpha-2) & $24(47 \%)$ & $24(46 \%)$ & $0(0 \%)$ & $19(83 \%)$ & $2(100 \%)$ & $\mathbf{0 . 0 0 8}$ \\
\hline RpA (D2, 5-HT1A) and RA (5-HT2A) & $7(14 \%)$ & $4(8 \%)$ & $0(0 \%)$ & $3(13 \%)$ & $2(100 \%)$ & $\mathbf{0 . 0 0 4}$ \\
\hline RA (D2, 5-HT2) and reuptake inhibitor (NET) & $6(12 \%)$ & $16(31 \%)$ & $1(50 \%)$ & $5(22 \%)$ & $1(50 \%)$ & 0.13 \\
\hline Mood stabilizers, mean (SD) & $0.3(0.5)$ & $0.2(0.5)$ & $0(0)$ & $0.3(0.4)$ & $0(0)$ & 0.88 \\
\hline Antidepressants, mean (SD) & $0.6(0.8)$ & $0.4(0.7)$ & $0(0)$ & $0.7(0.8)$ & $3(0)$ & $<$ \\
\hline Anxiolytics, mean (SD) & $0.7(0.8)$ & $0.6(0.8)$ & $0(0)$ & $1.3(1.2)$ & $1(0)$ & $<0.001$ \\
\hline Hypnotics, mean (SD) & $0.2(0.5)$ & $0.2(0.4)$ & $0(0)$ & $0.1(0.3)$ & $0(0)$ & $\mathbf{0 . 0 1 0}$ \\
\hline
\end{tabular}

Table 2: Differences between OA and NbN LAI classes.

RA: Receptorantagonist; RpA: Receptorpartialagonist.

\begin{tabular}{|c|c|c|c|c|c|c|}
\hline & oral only & RA (D2) & RA (D2, 5-HT2) & $\begin{array}{l}\text { RA (D2, 5-HT2, NE } \\
\text { alpha-2) }\end{array}$ & $\begin{array}{l}\text { RpA (D2, 5-HT1A) and } \\
\text { RA (5-HT2A) }\end{array}$ & $\mathrm{p}$-value \\
\hline $\mathrm{N}$ & 51 & 52 & 2 & 23 & 2 & \\
\hline Hospital admissions & $8(15.7 \%)$ & $12(23.1 \%)$ & $0(0 \%)$ & $11(47.8 \%)$ & $1(50 \%)$ & 0.036 \\
\hline Hospital admissions, days, mean (SD) & $4.2(11.8)$ & $11.3(34)$ & $0(0)$ & $22.3(36.8)$ & $22.5(31.8)$ & 0.12 \\
\hline Emergency Department visits, mean (SD) & $2.6(1.8)$ & $2(1.6)$ & $0(0)$ & $3.2(2.2)$ & $2(1.4)$ & 0.56 \\
\hline Occupation (having work) & $11(21.6 \%)$ & $3(5.8 \%)$ & $1(50 \%)$ & $4(17.4 \%)$ & $0(0 \%)$ & 0.102 \\
\hline
\end{tabular}

Table 4: Admission rate difference between OA and NbN LAI classes. 
Citation: Spínola C, Neto D, Santana L, Gago J (2021) Anxiolytic Medication and Long Acting Injectable Antipsychotics (LAI) in Schizophrenia: A Post-hoc Analysis of a 3-year Prospective Cohort Study. Int J Psychol Behav Anal 7: 172. doi: https://doi.org/10.15344/2455-3867/2021/172

Page 4 of 4

results, this argument should not be used to promote the introduction of LAI.

Regarding our primary outcome, hospital admissions, we found that patients in the OA group had fewer number and duration of admissions. The Receptor antagonist (D2) LAI class with the oldest LAI, is the only one to approach the OA values. A discussion about the efficacy of the newest LAI (at least risperidone and paliperidone, because the sample is too small to compare with aripiprazole) could be carried out, but we didn't have the sample size to allow it.

As expected, longer duration of illness showed a protective association against new admissions.

We also found a higher use of anxiolytic medication in the subgroup of risperidone and paliperidone. Is this an effect of these particular antipsychotics or a characteristic of the particular patients? We could argue that this association may be related to the side effects profile of these two related antipsychotics. Unfortunately, we did not include a screening tool regarding side effects in our study, and this question remains to be answered.

The prescription of anxiolytics at baseline showed a negative association with hospital admissions. Previous research showed ambivalent results with this therapeutic approach, probably because anxiolytic medication hides more profound problems, such as resistant positive symptoms, comorbid anxiety disorder, lack of employment, unstable and negative family and social relations, or the consequences of antipsychotic polypharmacy $[8,10]$. This group of patients should be further studied to understand the underpinnings of this association.

Our results evidence the need to implement non-pharmacological interventions focusing anxiety on patients with schizophrenia.

Our study has several limitations. The overall sample size is small and in some LAI classes impeditive of statistical analyses. In the inclusion criteria we considered patients with previous diagnosis of schizophrenia, and we did not assess the severity of positive and negative symptoms with standardized scales during the study. The absence of screening for side effects between groups is also a limitation. The study has the limitations regarding the chosen design. Larger and multicentric prospective cohorts are needed to clarify the results.

\section{Conclusions}

Our findings support that $\mathrm{OA}$ are more effective in preventing hospital admissions and duration of admission than LAI. The emergency department visits and occupation status showed no statistical association, but only a trend favoring OA regarding work. Being prescribed with anxiolytic medication is associated with a worst outcome regarding hospital admissions and this group of schizophrenic patients should be further investigated. Larger and multicentric prospective cohorts are needed to clarify the effects of particular classes of antipsychotics in the long-term prevention of admissions, symptomatology and remission. Anxiety and anxiolytics should be further investigated in schizophrenia.

\section{Competing Interests}

The authors declare that they have no competing interests.

\section{Author's Contributions}

Carla Spínola made contributions regarding conception and design, data collection and writing and revision of the manuscript.

Daniel Neto made contributions regarding conception and design, data analyses, writing and revision the manuscript.

Leonor Santana made contributions regarding conception and design, data collection and writing and revision of the manuscript.

Joaquim Gago made contributions regarding conception and design, writing and revision of the manuscript.

All authors gave final approval of the version to be published.

\section{Funding}

The authors declare that Carla Spínola was funded by the European Economic Area Grants to make part of the data collection of the patients, for an economical analyses published elsewhere [12].

\section{References}

1. Zohar J, Stahl S, Moller HJ, Blier P, Kupfer D, et al. (2015) A review of the current nomenclature for psychotropic agents and an introduction to the Neuroscience-based Nomenclature. Eur Neuropsychopharmacol 25: 23182325.

2. American Psychiatric Association (2013) Diagnostic and Statistical Manual of Mental Disorders. Fifth Edition.

3. Pelayo-Terán JM, Gajardo Galán VG, de la Ortiz-García de la Foz V, MartínezGarcía O, Tabarés-Seisdedos R, et al. Rates and predictors of relapse in firstepisode non-affective psychosis: a 3-year longitudinal study in a specialized intervention program (PAFIP). Eur Arch Psychiatry Clin Neurosci 267: 315323.

4. Heres S, Lambert M, Vauth R (2014) Treatment of early episode in patients with schizophrenia: the role of long acting antipsychotics. Eur Psychiatry 29: 1409-1413.

5. Braga R, Mendlowicz M, Marrocos R (2005) Anxiety disorders in outpatients with schizophrenia: prevalence and impact on the subjective quality of life. J Psychiatr Res 39: 409-414.

6. Huppert JD, Weiss KA, Lim R, Pratt S, Smith TE, et al. (2001) Quality of life in schizophrenia: Contributions of anxiety and depression. Schizophr Res 51: $171-180$.

7. Chakos MH, Glick ID, Miller AL, Hamner MB, Miller DD, et al. (2006) Special Section on CATIE Baseline Data: Baseline Use of Concomitant Psychotropic Medications to Treat Schizophrenia in the CATIE Trial. Psychiatr Serv 57: 1094-1101.

8. Chakos M, Patel J, Rosenheck R, Glick I, Hamner M, et al. (2011) Concomitant Psychotropic Medication Use During Treatment of Schizophrenia Patients: Longitudinal Results from the CATIE Study. Clin Schizophr Relat Psychoses 5: 124-134.

9. Ritsner MS, Gibel A (2006) The effectiveness and predictors of response to antipsychotic agents to treat impaired quality of life in schizophrenia: A 12-month naturalistic follow-up study with implications for confounding factors, antidepressants, anxiolytics, and mood stabilizers. Prog Neuropsychopharmacol Biol Psychiatry 30: 1442-1452.

10. Rubio JM, Correll CU (2017) Reduced all-cause mortality with antipsychotics and antidepressants compared to increased all-cause mortality with benzodiazepines in patients with schizophrenia observed in naturalistic treatment settings. Evid Based Ment Health 20: e6.

11. Fond G, Berna F, Boyer L, Godin O, Brunel L et al. (2018) Benzodiazepine long-term administration is associated with impaired attention/working memory in schizophrenia: results from the national multicentre FACE-SZ data set. Eur Arch Psychiatry Clin Neurosci 268: 17-26.

12. Matias MA, Chaves P, Pereira ME, Spinola C, Gago J, et al. (2017) Treatment Costs of Severe Mental Disorders in Portugal. J Ment Health Policy Econ. 